\title{
Refraction and scattering of radio emission from a solar source due to coronal inhomogeneities
}

\author{
Alexander N. Afanasiev, A. T. Altyntsev \\ Institute of Solar-Terrestrial Physics, P.O. Box 4026, 664033 Irkutsk, Russia \\ email: saf@iszf.irk.ru
}

\begin{abstract}
The paper presents study of the influence of coronal inhomogeneities on the average temporal pulse profile of radio emission from a compact solar pulse source located near the photosphere of the Sun. To consider both scattering and refraction of the radio emission propagating through the solar corona an integral representation of the wave field in the form of the interference integral is used. Thus formation of regular and random caustics and focuses as well as multipath structure of the radio emission received on the ground are taken into account. A numerical simulation of the average pulse profile, carried out for different parameters of the solar corona turbulence has shown that near-solar plasma can distort the emitted pulse significantly to form several pulses.
\end{abstract}

Recently a number of physical models have been proposed to interpret random intensity spikes of the solar radio emission, namely solar millisecond spike bursts. In most works devoted to solving this problem the main attention has been given to studying mechanisms of generation of the emission from sources located near the Sun's photosphere and to analysing statistics of these sources. Works on transformation of the solar radio emission along the propagation path through the solar corona have been presented to a much lesser extent. Meanwhile influence of the coronal regular and random inhomogeneities on characteristics of radio spike bursts may be considerable and even crucial in certain cases.

Let us represent the wave field at the receiver input as:

$$
u(t)=\int_{-\infty}^{\infty} Y(\omega) e^{i \omega t} d \omega
$$

where $\omega$ is the frequency, $Y(\omega)$ is the spectrum of the received signal.

For the average temporal pulse profile in the case of strong phase perturbations we can obtain:

$$
\bar{J} \equiv\left\langle u(t) u^{*}(t)\right\rangle=\iint_{-\infty}^{\infty} G\left(\omega_{1}\right) G^{*}\left(\omega_{2}\right) \Gamma_{\omega}\left(\omega_{1}, \omega_{2}\right) e^{i\left(\omega_{1}-\omega_{2}\right) t} d \omega_{1} d \omega_{2}
$$

here $\Gamma_{\omega}\left(\omega_{1}, \omega_{2}\right)$ is the frequency correlation function, $G(\omega)$ is the spectrum of the emitted signal, which we describe by a Gaussian function: $G(\omega)=\frac{T}{\sqrt{2 \pi}} \exp \left[-\frac{T^{2}}{2}\left(\omega-\omega_{0}\right)^{2}\right]$, with $T$ being the pulse duration and $\omega_{0}$ being the carrier frequency.

To determine the expression for the function $\Gamma_{\omega}\left(\omega_{1}, \omega_{2}\right)$ we should consider the trajectory problem of radio wave propagation in the solar corona. Calculations on the basis of the geometrical optics (GO) approximation has shown that a caustic peculiarity is formed even in the spherically symmetric solar corona. For describing the wave field in 


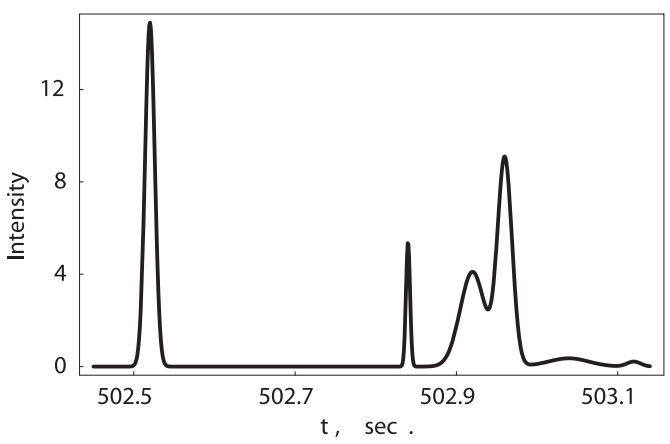

(a)

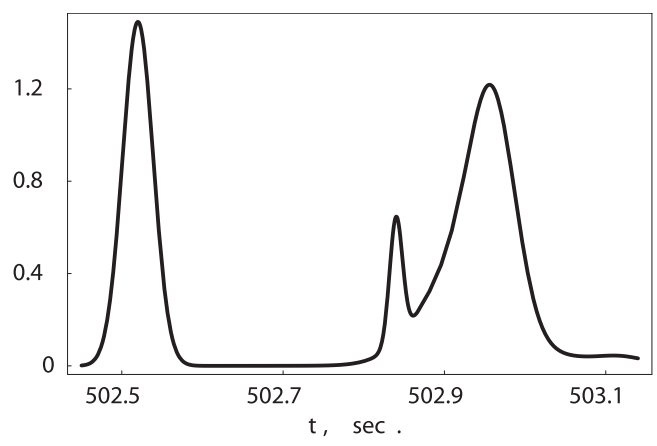

(b)

Figure 1. Average temporal pulse profile of the received signal from a pulse source located at the upper point of the coronal arch for the following values of the electron density disturbance: $0.01 \%(a), 0.1 \%(b) . T=10^{-4}$ sec.

the neighborhood of the caustic, the GO approximation is no longer applicable, and it is necessary to use geometrical optics generalizations, for example, the interference integral method. The ideology of this method implies that the wave field is represented as the integral sum of partial waves, each of which is characterized by the impact parameter. By determining $\Gamma_{\omega}\left(\omega_{1}, \omega_{2}\right)$ within the interference integral approximation and substituting it into (2) we obtain an expression for the average temporal pulse profile of the signal received on the Earth, which is valid in the neighborhood of caustic peculiarities.

We have carried out a numerical simulation of the average temporal pulse profile for different parameters of random near-solar plasma inhomogeneities in the presence of various large-scale coronal structures (coronal rays, transients, etc). As an example figure 1 presents the average pulse profiles of the solar source with initial Gaussian profile, located near an coronal arch for different values of the electron density disturbance. The arch is a large-scale structure with increased electron plasma density. We define its model as:

$$
N_{e}(r, \psi)=N_{0}(r)+N_{a r c} e^{-\left(\frac{r-r_{a r c}(\psi)}{L_{a r c}}\right)^{2}}
$$

where $r_{a r c}(\psi)=r_{\text {arc } 0}-\gamma\left(\psi-\psi_{\text {arc } 0}\right)^{2}$ and $\gamma=\left(r_{\text {arc } 0}-R_{s}\right) /\left(\psi_{s}-\psi_{\text {arc } 0}\right)^{2}$ Here $(r, \psi)$ are the polar coordinates, $N_{0}(r)$ is the regular profile of plasma electron density of the spherically symmetric solar corona, $\left(r_{\text {arc } 0}, \psi_{\text {arc } 0}\right)$ are the polar coordinates of the upper point of the arch, $R_{s}$ is the radius of the Sun's photosphere, $L_{\text {arc }}$ and $\left(\psi_{s}-\psi_{\text {arco }}\right)$ are radial and angular half-widths of the arch, respectively. As to scattering inhomogeneities we treat the near-solar plasma as a quasi-homogeneous random field and describe it by the power-law spectrum of inhomogeneities.

So, a short-time burst of radio emission arising near the photosphere of the Sun undergoes scattering due to turbulent coronal inhomogeneities. As a result the pulse suffers broadening and changing its initial shape. Regular refraction of radio emission on the large-scale coronal structures can result in forming regular caustics, focusing and defocusing radio waves and, thus, can distort the emitted pulse in addition. This work could be useful for interpreting the phenomenon of solar spike bursts.

\section{Acknowledgements}

This work was done under state support grant No. NSh-477.2003.2 for Leading Scientific Schools of the Russian Federation and the RFBR grant No. 03-02-16229. 